MATEC Web of Conferences 11,01023 (2014)

DOI: $10.1051 /$ matecconf / 20141101023

(C) Owned by the authors, published by EDP Sciences, 2014

\title{
Conception of a synthesis pozzolan from sediment dams calcined (Case: Gargar dams)
}

\author{
K. OUHBA ${ }^{1}$, L. BENAMARA ${ }^{1}$, A. HADJ HAMOUI ${ }^{1}$, A. HAMWI ${ }^{2}$ et MARIE - D. LOYE PILOT ${ }^{3}$ \\ ${ }^{1}$ Laboratoire d'Hydrologie et de Gestion des Ressources en eau Département d'Hydraulique, Université Des Sciences \\ et de la Technologie d'Oran Algérie.k_ouhbadz@yahoo.fr \\ ${ }^{2}$ Laboratoire des Matériaux Inorganiques Université Blaise Pascal Clermont-Ferrand France. \\ ${ }^{3}$ Centre d'Enseignement et de Recherche sur l'Environnement et les Sociétés, Ecole Normale Supérieure Paris, France
}

SUMMARY. Silting of dams is undoubtedly the most dramatic consequence of the erosion problem in Algeria. In spite of the great ways implemented for the construction of retaining structures, the storage of water remains confronted with the difficulties of their management. It is as important to be invested to prevent silting as to build a new dam.

There are two levels of Investigations: that is to say to prevent the solid matters from arriving in reserve. There, it is a question of putting obstacles to break the forces strength of surface waters but one can expect only partial results. The second solution is the evacuation of the sediments by a suitable management of the basic matters and desilting by dredging which requires the use of an external energy.

But considering the great quantity of the sediments of silting, we sought to make profitable the vases recovered with an aim of dumping down the costs associated with desilting. The research work undertaken herein concern the case of Gargar dam, as a real case of silted dams in Algeria and whose rate of silting is important. The significant amounts of rejected silts present environmental problems and their use as local raw materials for the manufacturing of materials in civil engineering contributes to its resolution.

The results of the physical and mineralogical characteristics of the sediments of Gargar dam directed us towards the design of a synthetic pozzolan. For, we carried out calcination of the silt at different temperatures up to $750{ }^{\circ} \mathrm{C}$ and prepared micro test samples of calcined silt-cement mixtures. The results show that the addition of $30 \%$ of sediments calcined at $750^{\circ} \mathrm{C}$ to cement permit to obtain a pozzolanic cement having similar characteristics with ordinary cement. This study shows therefore the possibility to value dam sediments as materials in the construction domain.

The analysis by the diffraction of x-rays (X-ray) and the differential thermal analysis (DTA) show that the chemical reactions between principal minerals of the vase used and cement give the formation of new products such as the freezing of HSC and the tobermorite.

Key words: dam - sediments - calcination - pozzolan - valorisation - matérials

Résumé. L'envasement des barrages est sans doute la conséquence la plus dramatique du problème de l'érosion en Algérie. Malgré les grands moyens mis en œuvre pour la construction des ouvrages de retenue, le stockage de l'eau reste confronté aux difficultés de leur gestion. Il est aussi important de s'investir à empêcher l'envasement que de construire un nouveau barrage. Les investigations se situent à deux niveaux : soit empêcher les matières solides d'arriver dans la retenue, là il s'agit de mettre des obstacles pour briser les forces vives des eaux de ruissellement mais on ne peut espérer que des résultats partiels. La deuxième solution est l'évacuation des sédiments par une gestion appropriée des organes de fond et le dévasement par dragage qui nécessite l'emploi d'une énergie externe. Mais devant les grandes quantités de sédiments d'envasement, nous avons jugé nécessaire de chercher à rentabiliser les vases récupérées dans le but d'amortir le coût lié au dévasement. Les travaux de recherches envisagés portent sur des cas réels de barrages envasés comme par exemple celui de Gargar (Algérie) dont le taux d'envasement est très important estimé à $30 \%$ de sa capacité initiale qui est de 450 millions de $\mathrm{m}^{3}$. Les quantités importantes de vases rejetées présentent un problème d'environnement, leur utilisation comme matières premières locales pour la fabrication des matériaux de génie civil contribue à sa résolution.

Les résultats des caractéristiques physiques et minéralogiques des sédiments de barrage de Gargar nous ont orientés vers la conception d'une pouzzolane de synthèse. Pour cela, on a procédé à la calcination de la vase à des températures différentes allant de $400^{\circ}$ jusqu'à $750^{\circ} \mathrm{C}$ et l'élaboration de micros éprouvettes de mélanges vase calcinée-ciment. Les résultats obtenus montrent que l'ajout de $30 \%$ de sédiments calcinés à $750^{\circ} \mathrm{C}$ au ciment, permet d'avoir un ciment pouzzolanique ayant des caractéristiques proches à un ciment ordinaire. Cette étude permet de valoriser les sédiments de barrages dans le domaine de matériaux.

Mots clé : barrage -Sédiments- calcination-pouzzolane- valorisation - matériaux 


\section{Introduction}

L'envasement des barrages en Algérie provient essentiellement de l'érosion des sols dans les bassins versants, suite aux pluies torrentielles qui se sont manifestées ces dernières années .Ce phénomène qui prend de l'ampleur surtout dans les régions pauvres en végétation, est la cause principale des quantités importantes de sédiments qui envahissent nos retenues et qu'on retrouve au fond des barrages en exploitation, causant principalement la diminution de la capacité utile. Ce phénomène entraîne une perte de potentiel allant jusqu'à annuler dans certains cas les fonctionnalités du barrage et peut présenter même des menaces pour l'environnement. C'est pour cette raison là qu'on est obligé de trouver une solution technique et économique pour évacuer et éliminer ces sédiments.

La diminution des potentialités hydrauliques de l'Algérie par suite de l'envasement des barrages est très importante, et l'opération de dévasement dans cette situation est la seule solution envisagée pour récupérer le volume perdu par l'envasement, mais cela demande beaucoup de moyens matériels et financiers. Pour subvenir à ces moyens, on doit envisager une solution d'utilisation de ces sédiments comme matières premières dans l'industrie des matériaux de génie civil ou autres, sous forme de vases récupérées lors des opérations de dévasement ou de vidange de fond.

Notre choix s'est basé sur les sédiments du barrage de Gargar situé à l'Ouest de l'Algérie, dans la wilaya de Relizane, mis en eau en 1988 avec une capacité initiale de 450 millions de mètres cube et dont le taux d'envasement est estimé à $30 \%$. Les essais d'identification ont montré que ces sédiments provenant des vidanges de fond sont des sols fins classés comme sol argileux très plastiques, composés suivant la courbe granulométrique essentiellement de sable fin, limon et argile.

$L$ 'analyse de diffractométrie aux rayons ' $X$ ' a montré l'existence de minéraux tels que le quartz, les carbonates et l'argile type kaolinite.

Des études antérieures ont montré que le traitement des sols argileux par la chaux ou le ciment ont donné de bons résultats au point de vue résistance à la compression pour éventuelle utilisation dans le domaine de génie civil, que ce soit dans leur état naturel brut ou traités $(4,7)$.

L'objectif de notre étude est de mettre en œuvre des solutions techniques suivant des critères économiques afin d'utiliser cette vase traitée thermiquement comme ajout pour jouer le rôle d'un ciment pouzzolanique d'un coté, et économiser l'énergie dans l'industrie de fabrication d'un ciment ordinaire.

\section{Identification des matériaux utilisés}

\subsection{Caractéristiques physiques.}

L'Analyse granulométrique nous a montré que cette vase est composée essentiellement de $25 \%$ de sable fin, $30 \%$ de limon et $45 \%$ d'argile (Fig. 1 )

Pour ce qui est des autres résultats on relève aussi que la densité des grains solides est évaluée à $26.0 \mathrm{KN} / \mathrm{m}^{3}$, la limite de liquidité $\mathrm{LL}=73 \%, \mathrm{LP}=27 \%$ et 1 'indice de plasticité $\mathrm{I}_{\mathrm{P}=46 \%}$.

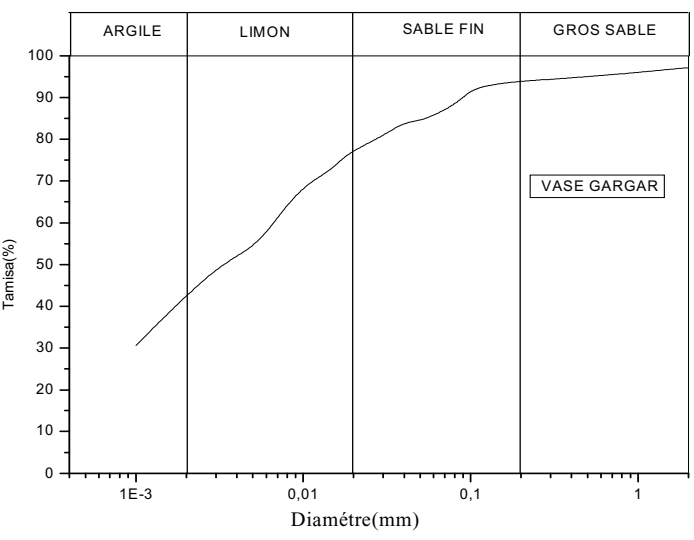

Figure $1:$ analyse granulométrique de la vase naturelle

D'après Les résultats obtenus à partir des analyses physiques, on constate que les sédiments du barrage Gargar sont des sols fins composés essentiellement d'Argile, sable fin et limon, et classés à partir de l'abaque de Casagrande comme sol argileux très plastiques. Ces analyses ont été réalisés au laboratoire de géotechnique de l'université de sciences et la technologie d'Oran « Algérie ».

\subsection{Analyses chimiques}

L'analyse chimique a été effectuée sur le produit réduit en poudre inférieur à 100 micromètres par fluorescence ' $\mathrm{X}$ ' au laboratoire de la cimenterie 'Lafarge' de Sig «Algérie». Les résultats obtenus montrent que le produit contient un pourcentage élevé en $\mathrm{SiO}_{2}$, suivi d'alumine $\left(\mathrm{Al}_{2} \mathrm{O}_{3}\right)$, le Cao et le $\mathrm{Fe}_{2} \mathrm{O}_{3}$ considérés comme principaux éléments de notre sol. (tableau.1). Le pourcentage de $\mathrm{CaCO}_{3}$ est estimé à $32.6 \%$.

Tableau.1 : Analyse chimique de la vase étudiée

\begin{tabular}{|c|c|}
\hline Eléments & $\%$ \\
\hline $\mathrm{SiO}_{2}$ & 44.12 \\
\hline $\mathrm{CaO}$ & 9.78 \\
\hline $\mathrm{Al}_{2} \mathrm{O}_{3}$ & 15.37 \\
\hline $\mathrm{Fe}_{2} \mathrm{O}_{3}$ & 6.10 \\
\hline $\mathrm{MgO}$ & 1.71 \\
\hline Perte au feu & 18.32 \\
\hline
\end{tabular}

\subsection{Analyse minéralogique.}

\subsubsection{Diffractions aux rayons ' $X$ '}

Cette analyse nous permet d'identifier les minéraux présents dans notre vase, elle a été réalisée au laboratoire de chimie inorganique de l'université de Blaise Pascal de Clermont- Ferrand, les diagrammes sont réalisés à l'aide d'un appareil X'pert ProMPD Philips.

L'analyse par diffractions aux rayons $\mathrm{X}$ de la vase à l'état naturel nous montre l'existence de raies correspondants aux minéraux tels que le quartz $\left(\mathrm{SiO}_{2}\right)$, les carbonates types calcite $\left(\mathrm{CaCO}_{3}\right)$, et les argiles type Kaolinite $\left(\mathrm{Al}_{2} \mathrm{O}_{3}\right.$ $\left.2 \mathrm{SiO}_{2} 2 \mathrm{H}_{2} \mathrm{O}\right)$ et l'illite, ainsi que la gibbsite $\mathrm{Al}(\mathrm{OH})_{3}$.

(Fig. 2). 


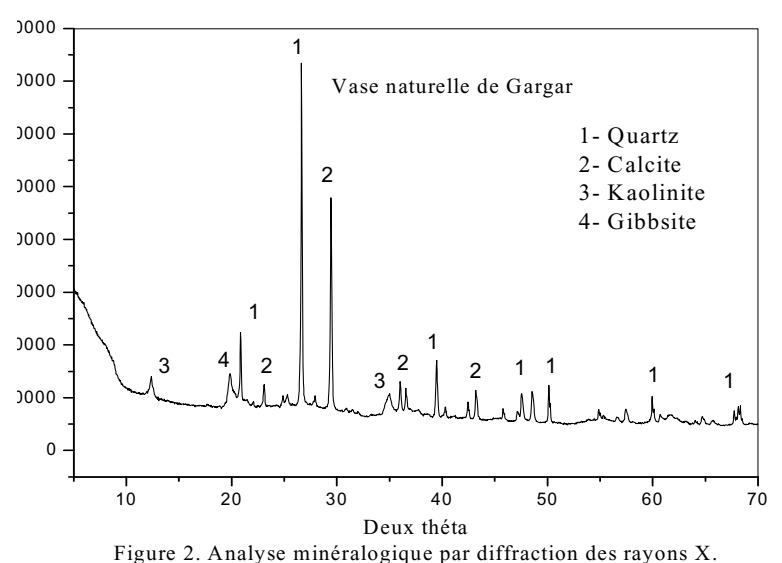

\subsubsection{Analyse thermogravimétrique}

Elle permet d'identifier les principaux minéraux par leurs pertes de poids, départ des hydroxydes $\mathrm{OH}^{-}$de liaison et du $\mathrm{CO}_{2}$. Les analyses ont été réalisées sur un appareil (TGA50 Shimadzu) au laboratoire de chimie inorganique de l'université de Blaise Pascal de Clermont-Ferrand.

D'après la courbe de l'analyse thermogravimétrique (ATG) effectuée sur la vase naturelle (Fig. 3), on note une perte de poids quasi linéaire jusqu'à $100^{\circ} \mathrm{C}$ correspondant au départ de l'eau absorbée, une petite variation entre $100^{\circ} \mathrm{C}$ et $420^{\circ} \mathrm{C}$ correspond à la perte d'eau de la gibbsite, une perte considérable entre $420^{\circ} \mathrm{C}$ et $640^{\circ} \mathrm{C}$ correspondant à l'élimination de l'eau de constitution de la Kaolinite et une chute quasi linéaire au-delà de $640^{\circ} \mathrm{C}$ correspondant au départ de $\mathrm{CO}_{2}$ des carbonates.

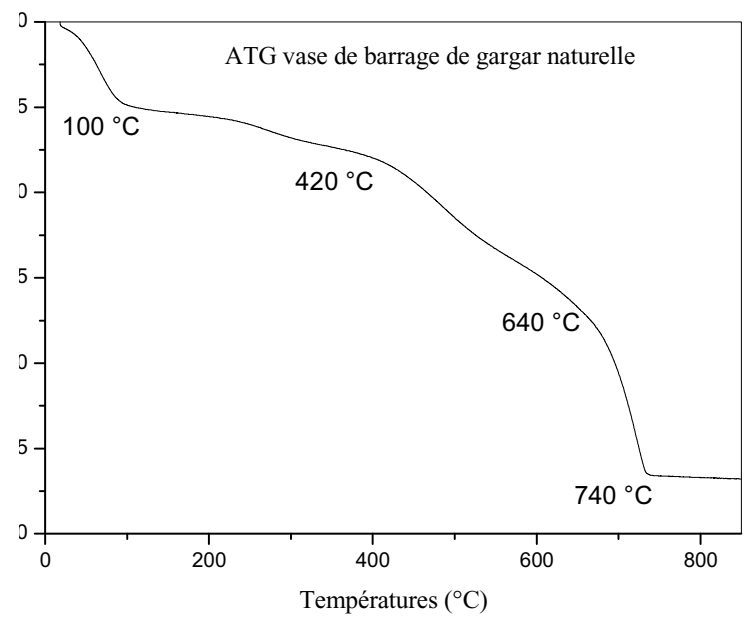

Figure 3: Analyse gravimétrie de la vase naturelle

\subsubsection{Analyse infrarouge (spectroscopie IR)}

C'est une analyse complémentaire aux analyses minéralogiques (DRX et ATG), elle permet d'identifier les principaux minéraux mal cristallisés dans un matériau argileux. Les analyses ont été réalisées au laboratoire de chimie inorganique de l'université de Blaise Pascal de Clermont-Ferrand.

Le spectre infrarouge de la vase naturelle (Fig. 4) montre essentiellement les bandes d'absorption du quartz raie à $1095 \mathrm{~cm}^{-1}$ et les carbonates raie à $1430 \mathrm{~cm}^{-1}$.

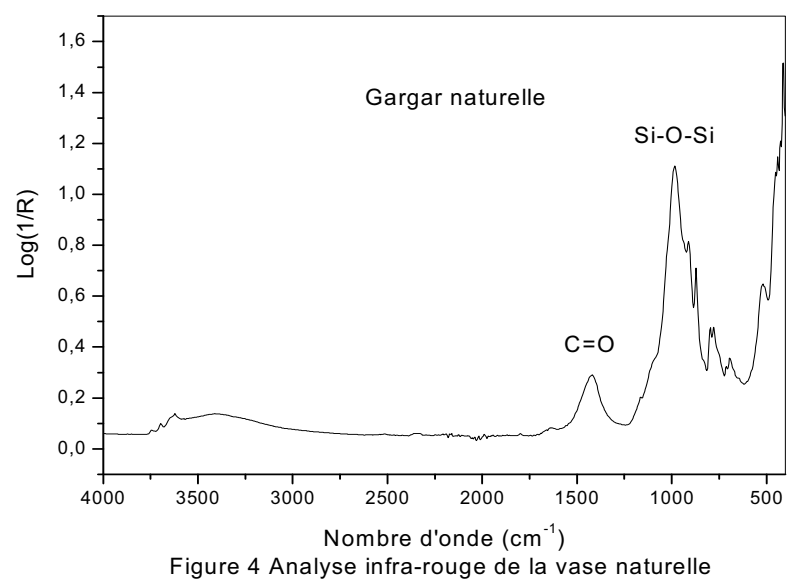

En conclusion, les analyses citées plus haut montrent en analyses minéralogiques l'existence des minéraux tels que le quartz, l'argile type Kaolinite et les carbonates type Calcite, et en analyses chimique la silice, l'alumine et l'oxyde de fer qui donnent à un sol sa forme pouzzolanique, ce qui nous permet de nous orienter vers l'axe de valorisation de notre vase de barrage en tant que pouzzolane de synthèse en la calcinant pour éliminer les carbonates. Cette vase dans son état calcinée peut être utilisée comme ajout au ciment 'CEMI', pour réduire le cout de production d'un ciment en économisant l'énergie, et diminuer la quantité de $\mathrm{CO}_{2}$ dégagée lors de la cuisson du clinker qui représente actuellement un grand problème pour le climat et l'environnement.

\section{Etude expérimentale}

\subsection{Traitement thermique}

En raison des études faites auparavant par différents auteurs quant à la réactivité des minéraux tels que les argiles en milieu normal de température et de pression $(1,8,11)$, et vue les résultats obtenus dans la caractérisation des sédiments du barrage de Gargar, nous procédons au traitement thermique par calcination des sédiments à une température optimale choisie parmi les différentes températures proposées afin d'avoir une meilleure calcination possible qui nous permet de gagner plus d'énergie et réduire l'émission du $\mathrm{CO}_{2}$ qui a un impact sur l'environnement (gaz à effet de serre) d'une part et d'autre part rendre la silice et l'alumine plus réactives avec la chaux libérée lors de l'hydratation du ciment.

Nous avons choisi des températures de calcination de 400, $550,650,700$ et $750^{\circ} \mathrm{C}$. La calcination de notre vase a été effectuée au laboratoire de métallurgie de l'université des sciences et la technologie d'Oran «Algérie ». Le produit cru est calciné dans un four électrique programmé : température de cuisson - durée de cuisson 5 heures montée de température $5^{\circ} \mathrm{C} /$ minute. On remarque d'après les résultats de diffraction aux rayons ' $X$ ', que la température de calcination pour laquelle le pic de la calcite ainsi que la kaolinite disparaissent complètement est de $750^{\circ} \mathrm{c}$ (Fig.5.E). Le traitement thermique transforme fortement la composition minéralogique du matériau, élimine les matières organiques et entraine la destruction des minéraux argileux par le départ de $\mathrm{H}_{2} \mathrm{O}$, si bien que 
des combinaisons amorphes apparaissent selon les réactions $(6,7)$.

$$
\begin{gathered}
\mathrm{CaCO}_{3} \stackrel{750^{\circ} \mathrm{c}}{\longrightarrow} \mathrm{CaO}+\mathrm{CO}_{2} \text { (gaz) } \\
\mathrm{Al}_{2} \mathrm{O}_{3}, 2 \mathrm{SiO}_{2}, 2 \mathrm{H}_{2} \mathrm{O} \stackrel{550-650^{\circ} \mathrm{c}}{\longrightarrow} \mathrm{Al}_{2} \mathrm{O}_{3} 2 \mathrm{SiO}_{2}+\mathrm{H}_{2} \mathrm{O} \text { (vap) }
\end{gathered}
$$

La possibilité de transformer les structures minérales de la vase de barrage dans le but d'en fabriquer des liants hydrauliques à usage courant, semble réalisable à condition que la teneur en argile soit suffisante. Par comparaison avec d'autres procédés, la vase doit être activée thermiquement afin de transformer les structures minérales qui sont à l'état naturel stable, en structures amorphes en raison de la fission des carbonates de calcium.

On constate d'après les résultats des différents diffractogrammes obtenus sur la vase calcinée à différentes températures, qu'à la température de $400^{\circ} \mathrm{C}$ (Fig.5. A) aucun changement ne se produit dans la structure minérale, à partir de $550^{\circ} \mathrm{C}$ (Fig.5. B) les pics correspondant aux argiles types kaolinites disparaissent et ceux des carbonates commencent à diminuer et disparaissent complètement à la température de $750^{\circ} \mathrm{C}$ (Fig.5 E). Les minéraux argileux sont détruits par le départ d'ions $\mathrm{OH}^{-}$ effets endothermiques vers $600^{\circ} \mathrm{C}$ et se reconstituent vers $1000^{\circ} \mathrm{C}$ effets exothermiques, tandis que les carbonates par le départ du $\mathrm{CO} 2$, cela veut dire que le traitement thermique transforme fortement la composition minéralogique du matériau. Des combinaisons amorphes apparaissent et la vase est transformée en pouzzolane de synthèse.

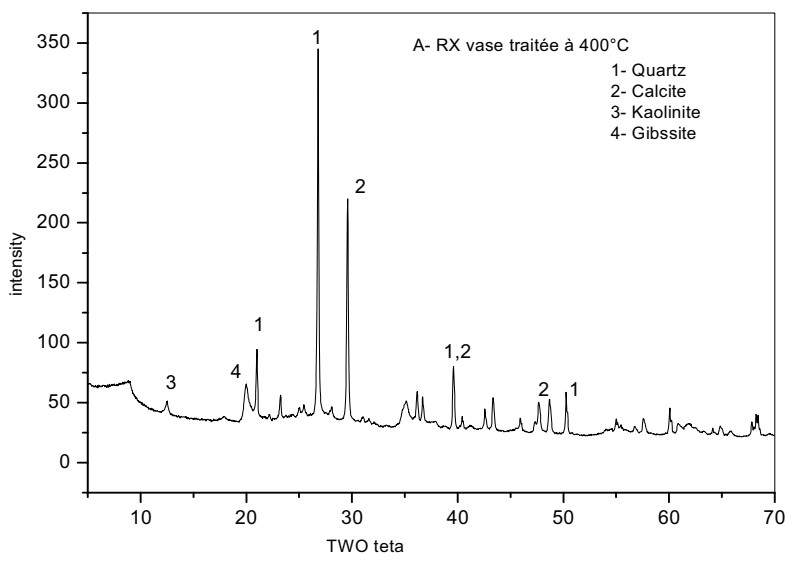

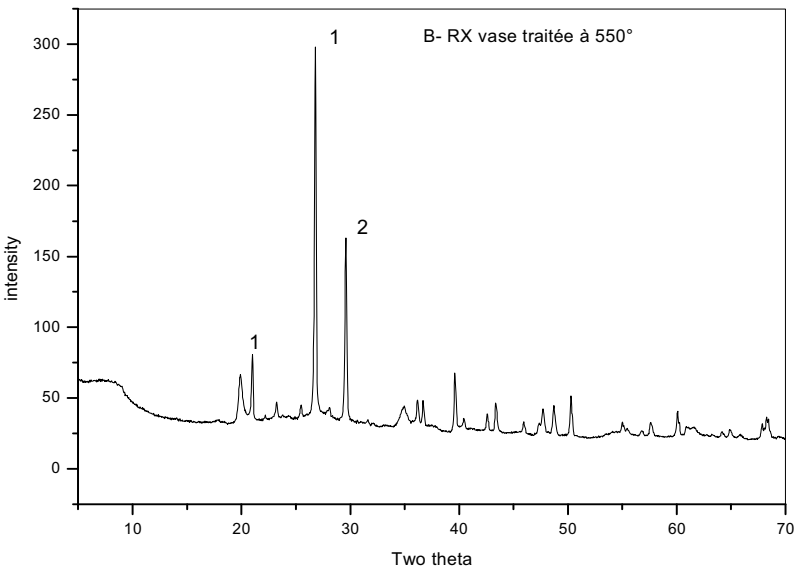
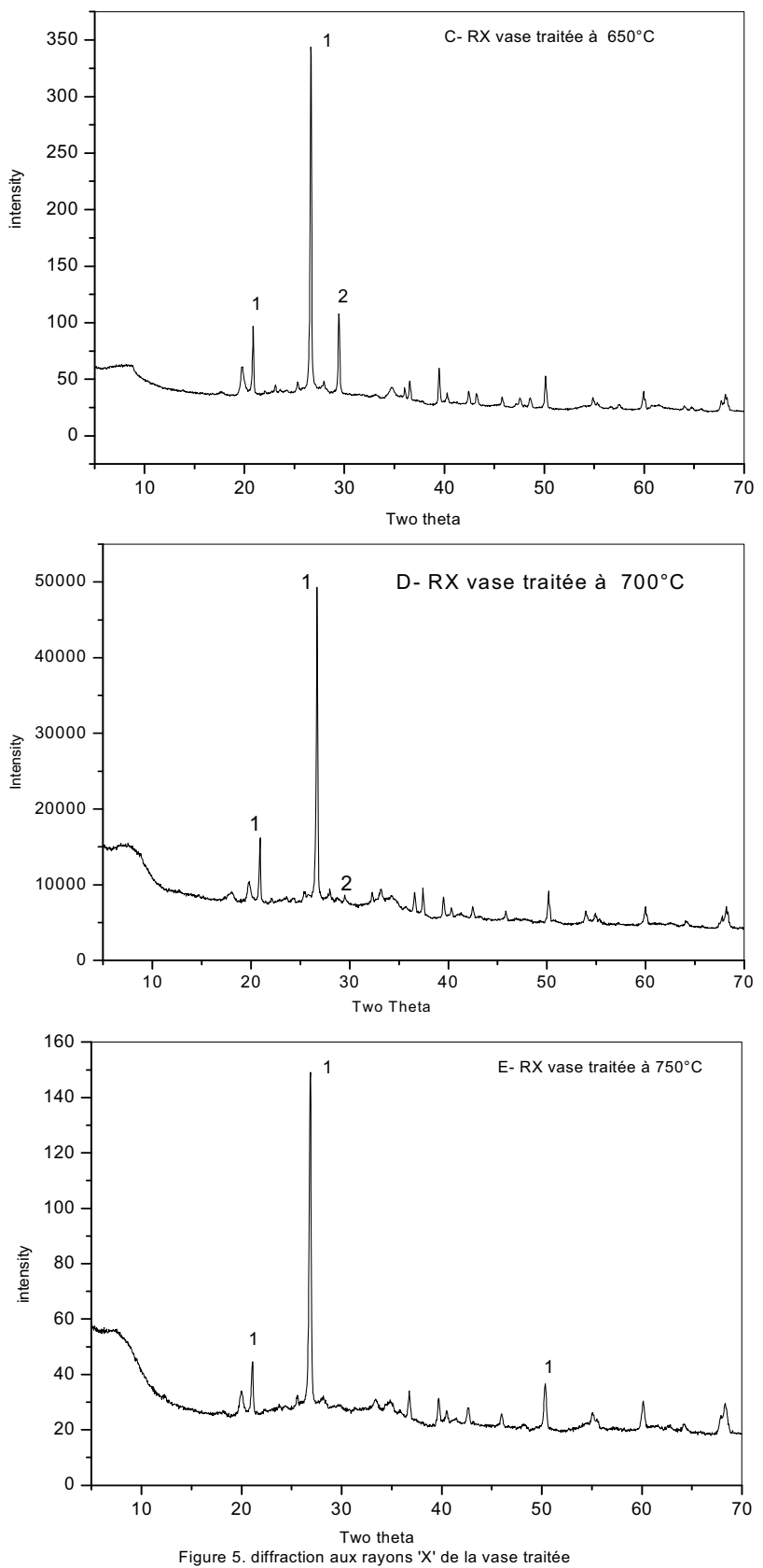


\subsection{Résultats des essais mécaniques}

Nous avons effectué des essais mécaniques sur un grand nombre d'éprouvettes pour chaque mélange (Fig.5) où on a constaté l'augmentation de la résistance mécanique à la compression en fonction de l'âge de conservation et le pourcentage en ciment. Le mélange composé de $70 \%$ de ciment et $30 \%$ de vase calcinée à $750^{\circ} \mathrm{C}$, présente des résistances mécaniques relativement élevées qui avoisinent celle du ciment (CEMI). Ces résistances sont les résultats de l'hydratation du ciment d'une part et la réaction pouzzolanique du mélange d'autre part.

La silice $\left(\mathrm{SiO}_{2}\right)$, l'alumine $\left(\mathrm{Al}_{2} \mathrm{O}_{3}\right)$ et l'oxyde de fer $\left(\mathrm{Fe}_{2} \mathrm{O}_{3}\right)$ se combinent avec la chaux $\mathrm{Ca}(\mathrm{OH})_{2}$ pour donner des gels de silicates de calcium hydratés CSH (type tobermorite) et d'aluminates de calcium hydratés CAH, tous deux responsables de la résistance. Les résultats des résistances peuvent être améliorés en ayant un temps de conservation plus long, sachant que la réaction pouzzolanique intervient après celle de l'hydratation.

Les réactions d'hydratation des ciments composés comprendront deux étapes. La première étape concernera l'hydratation du ciment (réaction I) et la deuxième étape concernera les réactions pouzzolaniques entre la chaux libérée par le ciment et les composants réactifs de la vase calcinée( réaction II).

Réaction d'hydratation (I)

$\mathrm{C}_{3} \mathrm{~S}+\mathrm{C}_{2} \mathrm{~S}+\mathrm{H}_{2} \mathrm{O}==>\mathrm{CxSHy}+\mathrm{CH}$ (portlandite)

Réaction pouzzolanique (II)

Vase calcinée + chaux $=\Rightarrow \mathrm{CxSHy}+\mathrm{C}_{2} \mathrm{ASH}_{8}+(\mathrm{m}-\mathrm{x}-2) \mathrm{CH}$

Le silicate d'aluminate de calcium hydraté $\mathrm{C}_{2} \mathrm{ASH}_{8}$ est instable en présence d'une solution saturée en chaux. Il donne le $\mathrm{CSH}$ et l'aluminate tétracalcique $\mathrm{C}_{3} \mathrm{AH}_{13}(6)$.

En conclusion, On peut dire qu'une partie de la chaux libérée du ciment a été consommée par la vase pour donner naissance à un gel de silicate de calcium hydraté type CSH non détectable par la diffraction des rayons X. $(4,7)$

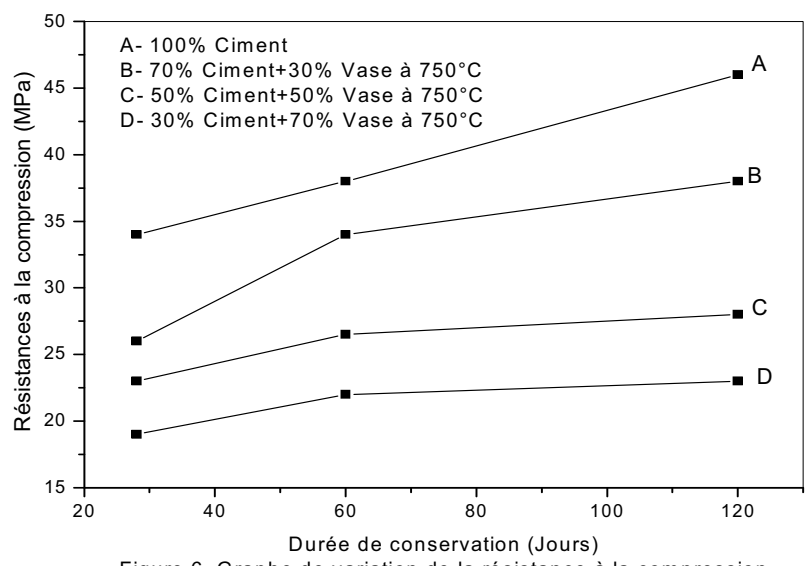

Figure 6. Graphe de variation de la résistance à la compression

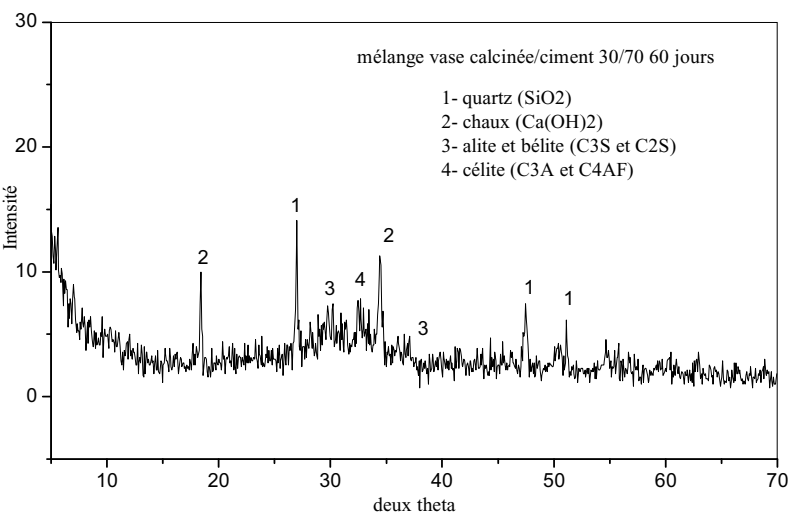

Figure 7. Diffractogramme des Rx mélange vase/ciment 30/70 à 60 jours

\section{Conclusion}

Les résultats obtenus des différents essais, nous ont permis de retenir que l'ajout de $30 \%$ de la vase calcinée présente une économie d'énergie et valorise les sédiments d'envasement des barrages longtemps considérés comme déchets. La vase calcinée, en se combinant à la chaux libérée par le ciment, donne naissance à un gel type tobermorite « $\mathrm{CSH} »$ mal cristallisé et non détectable par la diffractométrie des rayons $\mathrm{X}$. Une stabilisation thermique de ces sédiments est possible et une quantité de $30 \%$ de ciment peut-être substituée par la vase calcinée à $750^{\circ} \mathrm{C}$ pour avoir un ciment pouzzolanique. Cette stabilisation permet de transformer cette vase en pouzzolane de synthèse qui n'est pas le seul critère recherché.

La résistance mécanique augmente avec le pourcentage en ciment ajouté et avec l'âge de conservation. Le mélange à $30 \%$ de vase calcinée à $750{ }^{\circ} \mathrm{C}$ constitue un ciment pouzzolanique, puisqu'il conduit à des résistances en compression égales à $80 \%$ de celles obtenues avec le ciment seul. Des études sur la durabilité dans les milieux agressifs des matériaux composés à base de cette pouzzolane et de ciment portland peuvent être intéressantes.

\section{Références bibliographiques}

(1) K. OUHBA, L. BENAMARA. Réaction hydrothermale vase-ciment, revue trimestrielle internationale déchets sciences et techniques, ISSN 0753-3454, numéro 53, $1^{\mathrm{er}}$ trimestre 2009, pp 39-43

(2) L. BENAMARA, K. OUHBA. Stabilisation mécanique de la vase du cours d'eau d'El-Macta, le journal de l'eau et de l'environnement, revue semestrielle internationale scientifique et technique, ISSN 1112-3834, numéro 6, juin 2005, pp 18-23.

(3) K. OUHBA. Etude des propriétés des sédiments d'envasement et leur valorisation. "Cas de Fergoug et El Macta ", thèse de magister de l'Université de Sciences et de la Technologie d'Oran, Algérie, 2002.

(4) J. FRACHEBOURG. Contribution à l'étude de quelques Pouzzolanes naturelles et artificielles en vue de la fabrication des ciments de Pouzzolanes, Thèse Doctorat Es Sciences, Ecole Polytechnique, université de Lausanne $1965,136 \mathrm{p}$ 
(5) J. AMBROISE.Elaboration de liant pouzzolanique à moyenne température et étude de leurs propriétés physicochimiques.Thèse de DoctoratEs Sciences, INSA de Lyon $1984,167 \mathrm{p}$

(6) J. GNIEWEK. Réactivité des montmorillonites calcinées utilisation dans le Génie Civil, Thèse de Doctorat Es Sciences, INSA de Lyon 1987, 184 p.

(7) A. MESSI. Propriétés des ciments pouzzolaniques élaborés à partir de latérites activées thermiquement. Thèse de Doctorat, INSA de Lyon, 1988, 256 p.
(8) I.ROBU, R.JAUBERTINE .Etude diffractométrique de l'évolution des phases au cours du durcissement d'un ciment portland. Journal de physique ,2000,pp 473-479

(9) J.ABDO. Etude expérimentale de la stabilisation des arènes granitiques à la chaux. thèse de docteur .ing, univ.paris VI et ENSMines Paris. $1982,133 \mathrm{p}$

(10) BELL F.G. Lime stabilization of clay soils Bull.AIGI39, 1989, pp 67-74.

(11) AMARA KHAM-KHOEUP. Stabilisation à la chaux et au ciment des sédiments de l'Estuaire de la Rance, mémoire DEA, INSA de Rennes, 1996, 76 p 